\title{
The Chern Character on the Odd Spinor Groups
}

\author{
By
}

\author{
Takashi WATANABE*
}

\section{§1. Introduction}

This paper is a continuation of [10] and contains a calculation of the Chern character $([2, \S 1])$

$$
c h: K^{*}(G) \longrightarrow H^{*}(G ; Q)
$$

for $G=\operatorname{Spin}(2 l+1)$ with $l \geq 5$. For $G=\operatorname{Spin}(n)$ with $7 \leq n \leq 9$ it has been done in $[10]$.

Let $\lambda_{1}^{\prime}$ denote the composition

$$
\operatorname{Spin}(2 l+1) \stackrel{\pi}{\longrightarrow} S O(2 l+1) \stackrel{k}{\longrightarrow} S U(2 l+1) \stackrel{j}{\longrightarrow} U(2 l+1)
$$

where $\pi$ is the double covering, $k$ is the usual inclusion and $j$ is the natural inclusion. It gives an element of the representation ring $R$ $(\operatorname{Spin}(2 l+1))$, which we also denote by $\lambda_{1}^{\prime}$. Let

$$
\beta: R(\operatorname{Spin}(2 l+1)) \longrightarrow K^{-1}(\operatorname{Spin}(2 l+1))
$$

be the map introduced in [4]; then the image of $\beta$ generates $K^{*}$ (Spin $(2 l+1))$ multiplicatively. Since the $\lambda$-ring ([5, Chapter 12]) structure of $R(\operatorname{Spin}(2 l+1))$ is convenient for our work, if the value of $c h$ on $\beta\left(\lambda_{1}^{\prime}\right)$ is known, then that on any other element of $K^{*}(\operatorname{Spin}(2 l+1))$ can be calculated (see $\S 3$ ). For this reason the result below is the core of this paper. To state it we need some notations. For each integer $n$, let $s(n)$ be the integer such that

$$
2^{s(n)-1}<n \leq 2^{s(n)}
$$

Put

$$
\mathscr{P}=\left\{2^{i} \mid i \geq 0\right\} 。
$$

Recall that $H^{*}(\operatorname{Spin}(2 l+1) ; Q)$ is an exterior algebra on generators

Communicated by N. Shimada, October 14, 1985.

* Department of Mathematics, Osaka Women's University, Osaka 590, Japan. 
of degree $4 j-1,1 \leq j \leq l$. From this fact and the Poincaré duality we infer that there exist elements

$$
x_{4 j-1} \in H^{4 j-1}(\operatorname{Spin}(2 l+1) ; Z), 1 \leq j \leq l,
$$

such that (they are not divisible and) the product $x_{3} x_{70} \ldots x_{4 l-1}$ gives a generator of $H^{2 l^{2}+l}(\operatorname{Spin}(2 l+1) ; Z)=Z$.

Theorem 1. With the above notations, we have

$$
\operatorname{ch} \beta\left(\lambda_{1}^{\prime}\right)=\sum_{j=1}^{l} \frac{(-1)^{j-1} 2^{q(l, j)}}{(2 j-1) !} \cdot x_{4 j-1}
$$

where

$$
q(l, j)=\left\{\begin{array}{cc}
2 & \text { if } l \notin \mathscr{P} \text { and } j=2^{s(l)-1} \text { or } \\
& \quad l \in \mathscr{P} \text { and } j=2^{s(l)} \\
1 & \text { otherwise. }
\end{array}\right.
$$

As an application of this theorem, we obtain a precise description of the image of $x_{4 j-1}$ under the transgression in the Serre spectral sequence of the fibration $\operatorname{Spin}(2 l+1) \longrightarrow \operatorname{Spin}(2 l+1) / T \longrightarrow B T$, where $T$ is a maximal torus of $\operatorname{Spin}(2 l+1)$.

The paper is organized as follows. In $\S 2$ we determine the value of $k_{l}^{*}: H^{i}(\operatorname{Spin}(2 l+1) ; Z) \longrightarrow H^{i}(\operatorname{Spin}(2 l-1) ; Z)$ on $x_{4 j-1}$, where $k_{l}$ : $\operatorname{Spin}(2 l-1) \longrightarrow \operatorname{Spin}(2 l+1)$ is the natural inclusion. From the $\lambda$-ring structure of $R(\operatorname{Spin}(2 l+1))$ and a result of Atiyah [1], in $\S 3$ we derive a relation among the coefficients of $x_{4 j-1}$ 's in $\operatorname{ch} \beta\left(\lambda_{1}^{\prime}\right)$. In $\S 4$ we calculate the determinant of a certain matrix and then prove Theorem 1. Its consequences will be discussed in $\S 5$.

\section{§2. The Cohomology of $S \operatorname{pin}(2 l+1)$}

Let us begin by recalling some basic results on the cohomology of $\operatorname{Spin}(n)$ (for a reference see [3]). First,

(2.1) Spin(n) has 2-torsion if and only if $n \geq 7$, and all of its torsion is of order 2.

Secondly,

(2.2) If $p$ is an odd prime, then $H^{*}(\operatorname{Spin}(2 l+1) ; Z / p)$ is an exterior 
algebra on generators $x_{4 j-1}, 1 \leq j \leq l . \quad$ Each $x_{4 j-1}$ is universally transgressive.

Let $\Delta(\quad)$ denote a $Z / 2$-algebra having a simple system of generators. The following result is due to [7] (cf. [6]).

(2.3) (i) $H^{*}(\operatorname{Spin}(n) ; Z / 2)=\Delta\left(u_{i}, u \mid 0<i<n, i \notin \mathscr{P}\right)$ where deg $u_{i}=i$ and $\operatorname{deg} u=2^{s(n)}-1 . \quad u_{i}$ is universallv transgressive, and $u$ is universally transgressive if and only if $n \leq 9$.

(ii) $S q^{j}\left(u_{i}\right)=\left(\begin{array}{c}i \\ j\end{array}\right) u_{i+j}$; in particular, $u_{i}^{2}=u_{2 i}$ (where we use the convention that $u_{i}=0$ if $i \geq n$ or $\left.i \in \mathscr{P}\right) . S q^{1}(u)=0$ and $u^{2}=0$.

(iii) If $j_{n}: \operatorname{Spin}(n) \longrightarrow \operatorname{Spin}(n+1)$ is the natural inclusion, then $j_{n}^{*}\left(u_{i}\right)=u_{i}, j_{n}^{*}(u)=u$ if $n \notin \mathscr{P}$, and $j_{n}^{*}(u)=0$ if $n \in \mathscr{P}$.

Then we have

Lemma 2. Let $\rho: H^{i}(\operatorname{Spin}(2 l+1) ; Z) \longrightarrow H^{i}(\operatorname{Spin}(2 l+1) ; Z / 2)$ be the $\bmod 2$ reduction. Then for $1 \leq j \leq l$,

$$
\rho\left(x_{4 j-1}\right)= \begin{cases}u_{4 j-1} & \text { if } j \in \mathscr{P} \text { and } j<2^{s(2 l+1)-2} \\ u & \text { if } j=2^{s(2 l+1)-2} \\ u_{2 j-1} u_{2 j}+u_{4 j-1} & \text { if } j \notin \mathscr{P}\end{cases}
$$

(where deg $u=2^{s(2 l+1)}-1$ ).

Proof. Let $\left\{E_{r}\right\}$ be the mod 2 cohomology Bockstein spectral sequence of $\operatorname{Spin}(2 l+1)$. Since $E_{2}=E_{\infty}$ by (2.1), to prove this lemma, it suffices to compute $E_{2}=\operatorname{Ker} S q^{1} / \operatorname{Im} S q^{1}$. But it was done in Corollary 5 of [7] (by using (2.3) (ii)).

Consider the natural inclusion

$$
k_{l}=j_{2 l} j_{2 l-1}: \operatorname{Spin}(2 l-1) \longrightarrow \operatorname{Spin}(2 l+1)
$$

and put

$$
V_{l}=\operatorname{Spin}(2 l+1) / \operatorname{Spin}(2 l-1) .
$$

Then there is a fibration

$$
S^{2 l-1} \longrightarrow V_{l} \longrightarrow S^{2 l} \text {. }
$$

From the last statement of the theorem in $[9, \S 23.4]$ it follows that 
(2.4)

$$
\tilde{H}^{i}\left(V_{l} ; Z\right)=\left\{\begin{array}{cl}
Z & \text { if } i=4 l-1 \\
Z / 2 & \text { if } i=2 l \\
0 & \text { otherwise. }
\end{array}\right.
$$

Consider the homomorphism

$$
k_{l}^{*}: H^{i}(\operatorname{Spin}(2 l+1) ; Z) \longrightarrow H^{i}(\operatorname{Spin}(2 l-1) ; Z) .
$$

Glearly $k_{l}^{*}\left(x_{4 l-1}\right)=0$. We define integers $b_{j}(l)$ by the equations

$$
k_{l}^{*}\left(x_{4 j-1}\right)=b_{j}(l) \cdot x_{4 j-1}
$$

where $1 \leq j \leq l-1$. Note that

$$
s(2 l+1)= \begin{cases}s(l)+1 & \text { if } l \notin \mathscr{P} \\ s(l)+2 & \text { if } l \in \mathscr{P} .\end{cases}
$$

Lemma 3. We have, up to sign,

$$
b_{j}(l)= \begin{cases}2 & \text { if } l \in \mathscr{P} \text { and } j=2^{s(l)-1} \\ 1 & \text { otherwise. }\end{cases}
$$

Proof. Let $p$ be an odd prime, and consider the Serre spectral sequence for the $\bmod p$ cohomology of the fibration

$$
\operatorname{Spin}(2 l-1) \stackrel{{ }^{k_{l}}}{\longrightarrow} \operatorname{Spin}(2 l+1) \longrightarrow V_{l} \text {. }
$$

In view of (2.2) and (2.4), we see that it collapses. In other words,

$$
k_{l}^{*}: H^{i}(\operatorname{Spin}(2 l+1) ; Z / p) \longrightarrow H^{i}(\operatorname{Spin}(2 l-1) ; Z / p)
$$

is surjective for all $i$. On the other hand, by (2.3) (iii) we find that

$$
k_{l}^{*}: H^{i}(\operatorname{Spin}(2 l+1) ; Z / 2) \longrightarrow H^{i}(\operatorname{Spin}(2 l-1) ; Z / 2)
$$

is surjective if $l \notin \mathscr{P}$ (or $l \in \mathscr{P}$ and $i \neq 2^{s(l)+1}-1$ ). Therefore

$$
k_{l}^{*}: H^{i}(\operatorname{Spin}(2 l+1) ; Z) \longrightarrow H^{i}(\operatorname{Spin}(2 l-1) ; Z)
$$

is surjective if $l \notin \mathscr{P}$ (or $l \in \mathscr{P}$ and $i \neq 2^{s(l)+1}-1$ ). Hence $b_{j}(l)=1$ if $l \notin \mathscr{P}$ (or $l \in \mathscr{P}$ and $j \neq 2^{s(l)-1}$ ).

It remains to consider the case where $l \in \mathscr{P}$, i. e., $l=2^{s(l)}$. In this case, by (2.3) (iii) we find that

$$
u \in H^{2 l-1}(\operatorname{Spin}(2 l-1) ; Z / 2)
$$

is the only element which does not belong to the image of $k_{l}^{*}$. This implies that, in the integral cohomology spectral sequence of the above fibration, the only non-zero differential is 


$$
d_{2 l}(1 \otimes u)=v \otimes 1
$$

where $v$ is a generator of $H^{2 l}\left(V_{l} ; Z\right)=Z / 2$. By Lemma 2, this reveals that $k_{l}^{*}\left(x_{2 l-1}\right)=2 \cdot x_{2 l-1}$. That is, $b_{2^{s(l)-1}}\left(2^{s(l)}\right)=2$.

\section{§3. The Representation Ring of $S$ pirn $(2 \mathbb{Z}+\mathbb{1})$}

For further details of the following result, see [5] and [11].

(3. 1) (i) There exist representations $\lambda_{1}^{\prime}, \lambda_{2}^{\prime}, \ldots, \lambda_{l-1}^{\prime}, \Delta_{2 l+1}$ of $\operatorname{Spin}(2 l+1)$ such that

$$
R(\operatorname{Spin}(2 l+1))=Z\left[\lambda_{1}^{\prime}, \ldots, \lambda_{l-1}^{\prime}, \Delta_{2 l+1}\right]
$$

where $\operatorname{dim} \lambda_{k}^{\prime}=\left(\begin{array}{c}2 l+1 \\ k\end{array}\right), \operatorname{dim} \Delta_{2 l+1}=2^{l}$ and relations

$$
\begin{aligned}
& \Lambda^{k} \lambda_{1}^{\prime}=\lambda_{k}^{\prime}, \\
& \sum_{k=0}^{l} \lambda_{k}^{\prime}=\Delta_{2 l+1}^{2}
\end{aligned}
$$

hold (where $A^{k}$ denotes the $k$-th exterior power and we use the convention that $\lambda_{0}^{\prime}=1$ and $\left.\lambda_{l}^{\prime}=\Lambda^{l} \lambda_{1}^{\prime}\right)$.

(ii) The homomorphisin

$$
k_{l}^{*}: R(\operatorname{Spin}(2 l+1)) \longrightarrow R(\operatorname{Spin}(2 l-1))
$$

sends $\lambda_{1}^{\prime}$ to $\lambda_{1}^{\prime}+2$.

Consider now the composite

$$
R(\operatorname{Spin}(2 l+1)) \stackrel{\beta}{\longrightarrow} K^{-1}(\operatorname{Spin}(2 l+1)) \stackrel{c h}{\longrightarrow} H^{*}(\operatorname{Spin}(2 l+1) ; Q) .
$$

Since the cohomology suspension $\sigma^{*}: H^{i}(B \operatorname{Spin}(2 l+1) ; Q) \longrightarrow H^{i-1}$ $(\operatorname{Spin}(2 l+1) ; Q)$ has image contained in the module of primitives

$$
P H^{*}(\operatorname{Spin}(2 l+1) ; Q)=Q\left\{x_{4 j-1} \mid 1 \leq j \leq l\right\},
$$

it follows from the argument in $[10, \S 1]$ that, for any $\lambda \in R(\operatorname{Spin}$ $(2 l+1)), \operatorname{ch} \beta(\lambda)$ can be written in the form

$$
\operatorname{ch} \beta(\lambda)=\sum_{j=1}^{l} a(\lambda, j) \cdot x_{4 j-1} \text { with } a(\lambda, j) \in Q .
$$

For clarity we sometimes write $\lambda_{k}^{\prime}(l)$ for $\lambda_{k}^{\prime} \in R(\operatorname{Spin}(2 l+1))$.

Lemma 4. For $1 \leq j \leq l-1$,

$$
a\left(\lambda_{1}^{\prime}(l), j\right)= \begin{cases}2^{-1} a\left(\lambda_{1}^{\prime}(l-1), j\right) & \text { if } l \in \mathscr{P} \text { and } j=2^{s(l)-1} \\ a\left(\lambda_{1}^{\prime}(l-1), j\right) & \text { otherwise. }\end{cases}
$$


Proof. By (3.2),

$$
\operatorname{ch} \beta\left(\lambda_{1}^{\prime}(l)\right)=\sum_{j=1}^{l} a\left(\lambda_{1}^{\prime}(l), j\right) x_{4 j-1} .
$$

Apply $k_{l}^{*}$ to this equation. Then, by (3.1)(ii) and the fact that $\beta(2)=0([4$, Lemma 4.1$])$, the left hand side becomes

$$
\begin{aligned}
k_{l}^{*} \operatorname{ch} \beta\left(\lambda_{1}^{\prime}(l)\right) & =\operatorname{ch} \beta k_{l}^{*}\left(\lambda_{1}^{\prime}(l)\right) \\
& =\operatorname{ch} \beta\left(\lambda_{1}^{\prime}(l-1)\right) \\
& =\sum_{j=1}^{l-1} a\left(\lambda_{1}^{\prime}(l-1), j\right) x_{4 j-1}
\end{aligned}
$$

and the right hand side becomes

$$
\begin{aligned}
k_{l}^{*}\left(\sum_{j=1}^{l} a\left(\lambda_{1}^{\prime}(l), j\right) x_{4 j-1}\right) & =\sum_{j=1}^{l} a\left(\lambda_{1}^{\prime}(l), j\right) k_{l}^{*}\left(x_{4 j-1}\right) \\
& =\sum_{j=1}^{l-1} a\left(\lambda_{1}^{\prime}(l), j\right) b_{j}(l) x_{4 j-1} .
\end{aligned}
$$

Hence

$$
a\left(\lambda_{1}^{\prime}(l-1), j\right)=a\left(\lambda_{1}^{\prime}(l), j\right) \cdot b_{j}(l)
$$

for $1 \leq j \leq l-1$. So the result follows from Lemma 3 .

Let us review a result of Atiyah [1]. By (3. 1) (i), the set $\left\{\lambda_{1}^{\prime}, \ldots\right.$, $\left.\lambda_{l-1}^{\prime}, \Delta_{2 l+1}\right\}$ is a system of generators of the ring $R(\operatorname{Spin}(2 l+1))$. Via (3.2), with such a system there is associated an $l \times l$-matrix

$$
\Phi(l)=\left(\begin{array}{ccc}
a\left(\lambda_{1}^{\prime}, 1\right) & \ldots & a\left(\lambda_{1}^{\prime}, l\right) \\
\vdots & & \vdots \\
a\left(\lambda_{l-1}^{\prime}, 1\right) & \ldots & a\left(\lambda_{l-1}^{\prime}, l\right) \\
a\left(\Delta_{2 l+1}, 1\right) & \ldots & a\left(\Delta_{2 l+1}, l\right)
\end{array}\right) .
$$

Then his result (Proposition 1 of [1]) can be reformulated as det $\Phi(l)= \pm 1$ (see $[10, \S 0])$. Here we adopt

$$
\operatorname{det} \Phi(l)=1
$$

only, because the problem of sign (e.g., the sign of $x_{4 j-1}$ and the arrangement of $x_{4 j-1}$ 's) has no essential influence on our argument.

In what follows we express $a\left(\lambda_{k}^{\prime}, j\right)$ and $a\left(\Delta_{2 l+1}, j\right)$ in terms of $a\left(\lambda_{1}^{\prime}, j\right)$. First, by Lemma 1 of $[10]$, we deduce from the relation (a) in (3.1) (i) that

$$
a\left(\lambda_{k}^{\prime}, j\right)=\varphi(2 l+1, k, 2 j) \cdot a\left(\lambda_{1}^{\prime}, j\right)
$$

for all $k \geq 1$ where 


$$
\varphi(2 l+1, k, 2 j)=\sum_{i=1}^{k}(-1)^{i-1}\left(\begin{array}{c}
2 l+1 \\
k-i
\end{array}\right) i_{2 j-1} .
$$

Consider next the relation (b) in (3.1)(i). Since $\beta$ is additive and $\beta(1)=0$, we have

$$
\begin{aligned}
\operatorname{ch} \beta\left(\sum_{k=0}^{l} \lambda_{k}^{\prime}\right) & =\sum_{k=0}^{l} \operatorname{ch} \beta\left(\lambda_{k}^{\prime}\right)=\sum_{k=1}^{l} \operatorname{ch} \beta\left(\lambda_{k}^{\prime}\right) \\
& =\sum_{k=1}^{l} \sum_{j=1}^{l} \varphi(2 l+1, k, 2 j) a\left(\lambda_{1}^{\prime}, j\right) x_{4 j-1} .
\end{aligned}
$$

Using the formula (2) of $[4, \mathrm{p} .8]$, we have

$$
\begin{aligned}
\operatorname{ch} \beta\left(\Delta_{2 l+1}^{2}\right) & =\operatorname{ch}\left\{2^{l} \beta\left(\Delta_{2 l+1}\right)+2^{l} \beta\left(\Delta_{2 l+1}\right)\right\} \\
& =2^{l+1} \operatorname{ch} \beta\left(\Delta_{2 l+1}\right) .
\end{aligned}
$$

Therefore

$$
a\left(\Delta_{2 l+1}, j\right)=2^{-(l+1)}\left\{\sum_{k=1}^{l} \varphi(2 l+1, k, 2 j)\right\} \cdot a\left(\lambda_{1}^{\prime}, j\right) .
$$

Combining the above, we see that

$$
\operatorname{det} \Phi(l)=2^{-(l+1)} \cdot \operatorname{det}\left(\begin{array}{c}
\Phi_{1}(l) \\
\vdots \\
\Phi_{l-1}(l) \\
\sum_{k=1}^{l} \Phi_{k}(l)
\end{array}\right) \cdot \prod_{j=1}^{l} a\left(\lambda_{1}^{\prime} j\right)
$$

where $\Phi_{k}(l)$ is the $1 \times l$-matrix $(\varphi(2 l+1, k, 2 j))_{1 \leq j \leq l \cdot}$ By elementary properties of determinant,

$$
\operatorname{det}\left(\begin{array}{c}
\Phi_{1}(l) \\
\vdots \\
\Phi_{l-1}(l) \\
\sum_{k=1}^{l} \Phi_{k}(l)
\end{array}\right)=\sum_{k=1}^{l} \operatorname{det}\left(\begin{array}{c}
\Phi_{1}(l) \\
\vdots \\
\Phi_{l-1}(l) \\
\Phi_{k}(l)
\end{array}\right)=\operatorname{det}\left(\begin{array}{c}
\Phi_{1}(l) \\
\vdots \\
\Phi_{l-1}(l) \\
\Phi_{l}(l)
\end{array}\right)
$$

Therefore

$$
2^{l+1} \cdot \operatorname{det} \Phi(l)=\operatorname{det}(\varphi(2 l+1, i, 2 j))_{1 \leq i, j \leq l} \circ \prod_{j=1}^{l} a\left(\lambda_{1}^{\prime}, j\right) .
$$

Substituting (3.3) to this, we get

$$
\prod_{j=1}^{l} a\left(\lambda_{1}^{\prime}, j\right)=2^{l+1} / \operatorname{det}(\varphi(2 l+1, i, 2 j))_{1 \leq i, j \leq l^{\circ}}
$$

\section{§4. Proof of Theorem $\mathbb{1}$}

The determinant in (3.4) is given by 
Lemma 5. $\operatorname{det}(\varphi(2 l+1, i, 2 j))_{1 \leq i, j \leq l}=(-1)^{[l / 2]} \prod_{k=1}^{l}(2 k-1) !$ where $[l / 2]$ denotes the greatest integer less than or equal to $l / 2$.

Proof. For $k-1 \leq i \leq l$ and $k \leq j \leq l$, let

$$
\varphi_{i, j}^{(k)}=\sum_{m=k}^{i}(-1)^{m-1}\left(\begin{array}{c}
2 l+1 \\
i-m
\end{array}\right)\left(m^{2}-1\right)\left(m^{2}-2^{2}\right) \ldots\left(m^{2}-(k-1)^{2}\right) m^{2 j-2 k+1}
$$

Note that when $k=1, \varphi_{i, j}^{(1)}=\varphi(2 l+1, i, 2 j)$. As is easily checked, these integers satisfy:

$$
\begin{aligned}
& \varphi_{k-1, j}^{(k)}=0 \quad \text { for } k \leq j \leq l ; \\
& \varphi_{k, k}^{(k)}=(-1)^{k-1}(2 k-1) ! ; \\
& \varphi_{k, j}^{(k)}=k^{2} \varphi_{k, j-1}^{(k)} \quad \text { for } k+1 \leq j \leq l .
\end{aligned}
$$

Furthermore, if $k \leq i \leq l$ and $k+1 \leq j \leq l$, then

$$
\begin{aligned}
& \varphi_{i, j}^{(k)}-k^{2} \varphi_{i, j-1}^{(k)} \\
& =\sum_{m=k}^{i}(-1)^{m-1}\left(\begin{array}{c}
2 l+1 \\
i-m
\end{array}\right)\left(m^{2}-1\right) \ldots\left(m^{2}-(k-1)^{2}\right)\left(m^{2 j-2 k+1}-k^{2} m^{2 j-2 k-1}\right) \\
& =\sum_{m=k+1}^{i}(-1)^{m-1}\left(\begin{array}{c}
2 l+1 \\
i-m
\end{array}\right)\left(m^{2}-1\right) \ldots\left(m^{2}-(k-1)^{2}\right)\left(m^{2}-k^{2}\right) m^{2 j-2(k+1)+1} \\
& =\varphi_{i, j}^{(k+1)} .
\end{aligned}
$$

For each $k$ with $1 \leq k \leq l$, we consider the $(l-k+1) \times(l-k+1)-$ matrix $\left(\varphi_{i, j}^{(k)}\right)_{k \leq i, j \leq l}$. Transform this matrix by adding $\left(-k^{2}\right)$-times the $(l-k)$-th column to the $(l-k+1)$-th column. Transform next the resulting matrix by adding $\left(-k^{2}\right)$-times the $(l-k-1)$-th column to the $(l-k)$-th column. Iterate such elementary transformations. This procedure, which ends in the first column, yields the following first equality:

$$
\operatorname{det}\left(\varphi_{i, j}^{(k)}\right)=\operatorname{det}\left(\begin{array}{cc}
\varphi_{k, k}^{(k)} & 0 \\
\varphi_{i, k}^{(k)} & \varphi_{i, j}^{(k+1)}
\end{array}\right)=\varphi_{k, k}^{(k)} \operatorname{det}\left(\varphi_{i, j}^{(k+1)}\right) .
$$

So the $(l-k) \times(l-k)$-matrix $\left(\varphi_{i, j}^{(k+1)}\right)_{k+1 \leq i, j \leq l}$ is left. From this recursive process we deduce that

$$
\begin{aligned}
\operatorname{det}\left(\varphi_{i, j}^{(1)}\right)_{1 \leq i, j \leq l} & =\prod_{k=1}^{l} \varphi_{k, k}^{(k)} \\
& =\prod_{k=1}^{l}(-1)^{k-1}(2 k-1) ! \\
& =(-1)^{[l / 2]} \prod_{k=1}^{l}(2 k-1) ! .
\end{aligned}
$$


By this lemma and (3.4),

$$
\prod_{j=1}^{l} a\left(\lambda_{1}^{\prime}, j\right)=(-1)^{[l / 2]} 2^{l+1} / \prod_{k=1}^{l}(2 k-1) ! .
$$

The following is a restatement of Theorem 1 .

Theorem 6. For $1 \leq j \leq l$,

$$
a\left(\lambda_{1}^{\prime}(l), j\right)=\left\{\begin{array}{lc}
-2^{2} /(2 j-1) ! & \text { if } l \notin \mathscr{P} \text { and } j=2^{s(l)-1} \text { or } \\
& l \in \mathscr{P} \text { and } j=2^{s(l)} \\
(-1)^{j-1} 2 /(2 j-1) ! & \text { otherwise. }
\end{array}\right.
$$

Proof. We prove this by induction on $l$. The result for $l=3,4$ was shown in $[10, \S 3]$. Assume that it is true for $l=m$ and consider the case $l=m+1$. Our argument is divided into two cases. (Recall that $2^{s(m)-1}<m \leq 2^{s(m)}$.)

First suppose that $m+1 \notin \mathscr{P}$. By Lemma 4 and the inductive hypothesis, we have, for $1 \leq j \leq m$,

$$
\begin{aligned}
& a\left(\lambda_{1}^{\prime}(m+1), j\right)=a\left(\lambda_{1}^{\prime}(m), j\right) \\
& =\left\{\begin{array}{lc}
-2^{2} /(2 j-1) ! & \text { if } m \notin \mathscr{P} \text { and } j=2^{s(m)-1} \text { or } \\
& m \in \mathscr{P} \text { and } j=2^{s(m)} \\
(-1)^{j-1} 2 /(2 j-1) ! & \text { otherwise. }
\end{array}\right.
\end{aligned}
$$

If $m \notin \mathscr{P}$, then $s(m+1)=s(m)$. If $m \in \mathscr{P}$, then $m=2^{s(m)}$ and so $s(m+1)$ $=s(m)+1$. Thus the desired result is obtained for $j \neq m+1$. Using the above and (4.1), we have

$$
\begin{aligned}
a\left(\lambda_{1}^{\prime}(m+1), m+1\right) & =\left\{\prod_{j=1}^{m+1} a\left(\lambda_{1}^{\prime}(m+1), j\right)\right\} /\left\{\prod_{j=1}^{m} a\left(\lambda_{1}^{\prime}(m+1), j\right)\right\} \\
& =\left\{\prod_{j=1}^{m+1} a\left(\lambda_{1}^{\prime}(m+1), j\right)\right\} /\left\{\prod_{j=1}^{m} a\left(\lambda_{1}^{\prime}(m), j\right)\right\} \\
& =\frac{\left\{(-1)^{[(m+1) / 2]} 2^{m+2} / \prod_{j=1}^{m+1}(2 j-1) !\right\}}{\left\{(-1)^{[m / 2]} 2^{m+1} / \prod_{j=1}^{m}(2 j-1) !\right\}} \\
& =(-1)^{[(m+1) / 2]-[m / 2]} 2 /(2 m+1) ! \\
& =(-1)^{m} 2 /(2 m+1) !
\end{aligned}
$$

Finally suppose that $m+1 \in \mathscr{P}$. Then $m+1=2^{s(m)}$ and $s(m+1)=$ $s(m)$. By Lemma 4 and the inductive hypothesis, we have, for $1 \leq j \leq m$, 


$$
\begin{aligned}
a\left(\lambda_{1}^{\prime}(m+1), j\right) & = \begin{cases}2^{-1} a\left(\lambda_{1}^{\prime}(m), j\right) & \text { if } j=2^{s(m+1)-1} \\
a\left(\lambda_{1}^{\prime}(m), j\right) & \text { otherwise }\end{cases} \\
& = \begin{cases}2^{-1}\left(-2^{2}\right) /(2 j-1) ! & \text { if } j=2^{s(m)-1} \\
(-1)^{j-1} 2 /(2 j-1) ! & \text { otherwise }\end{cases} \\
& =(-1)^{j-1} 2 /(2 j-1) ! .
\end{aligned}
$$

Thus the desired result is obtained for $j \neq m+1$. Using the above and (4.1), we have

$$
\begin{aligned}
a\left(\lambda_{1}^{\prime}(m+1), m+1\right) & =\left\{\prod_{j=1}^{m+1} a\left(\lambda_{1}^{\prime}(m+1), j\right)\right\} /\left\{\prod_{j=1}^{m} a\left(\lambda_{1}^{\prime}(m+1), j\right)\right\} \\
& =\left\{\prod_{j=1}^{m+1} a\left(\lambda_{1}^{\prime}(m+1), j\right)\right\} /\left\{\prod_{j=1}^{m}(-1)^{j-1} 2 /(2 j-1) !\right\} \\
& =\left\{2^{m+2} / \prod_{j=1}^{m+1}(2 j-1) !\right\} /\left\{-2^{m} / \prod_{j=1}^{m}(2 j-1) !\right\} \\
& =-2^{2} /(2 m+1) !
\end{aligned}
$$

and the proof is completed.

\section{§5. Some Consequences}

The argument of this section depends on that of $[10, \S 1]$; the reader is referred to it.

A maximal torus $T$ of $\operatorname{Spin}(2 l+1)$ can be chosen so that

$$
H^{*}(B T ; Z)=Z\left[t_{1}, t_{2}, \ldots, t_{l}, \gamma\right] /\left(c_{1}-2 \gamma\right)
$$

where $\operatorname{deg} t_{j}=\operatorname{deg} \gamma=2$ and $c_{1}=t_{1}+\cdots+t_{l}$. Moreover, if $W(\operatorname{Spin}(2 l+$ 1)) is the corresponding Weyl group, then it acts on $H^{*}(B T ; Z)$ and the $W(\operatorname{Spin}(2 l+1))$-invariants in $H^{*}(B T ; Q)$ form a polynomial algebra on generators $p_{1}, p_{2}, \ldots, p_{l}$ where

$$
p_{j}=\sigma_{j}\left(t_{1}^{2}, t_{2}^{2}, \ldots, t_{l}^{2}\right) \in H^{4 j}(B T ; Z)
$$

( $\sigma_{j}$ denotes the $j$-th elementary symmetric function). Let $i: T \rightarrow S p i n$ $(2 l+1)$ be the inclusion and $i^{*}: R(\operatorname{Spin}(2 l+1)) \longrightarrow R(T)$ the homomorphism induced by it. Let $\alpha: R(T) \longrightarrow K^{*}(B T)$ be the ( $\lambda$-ring) homomorphism given in [2]. For $k \geq 0$ let $c h^{k}: K^{*}(B T) \longrightarrow H^{2 k}(B T ; Q)$ be the composition $K^{*}(B T) \stackrel{c h}{\longrightarrow} H^{*}(B T ; Q) \longrightarrow H^{2 k}(B T ; Q)$ where the second map is the projection onto the $2 k$-dimensional component. Consider the cohomology transgression

$$
\tau^{\prime}: H^{i-1}(\operatorname{Spin}(2 l+1) ; Q) \longrightarrow H^{i}(B T ; Q)
$$


in the Serre spectral sequence of the fibration

$$
\operatorname{Spin}(2 l+1) \longrightarrow \operatorname{Spin}(2 l+1) / T \longrightarrow B T .
$$

Then we have

Proposition 7. With the above notations, for $1 \leq j \leq l$,

$$
\tau^{\prime}\left(x_{4 j-1}\right)=2^{-q(l, j)} p_{j}
$$

(modulo decomposables in $Q\left[p_{1}, p_{2}, \ldots, p_{l}\right]$ ).

Proof. Since the set of weights of $\lambda_{1}^{\prime}$ is given by $\left\{ \pm t_{j}, 0 \mid 1 \leq j \leq l\right\}$ (see [11]), it follows from the same calculation as in $[10, \S 2]$ that

$$
c h^{2 j} \alpha i^{*}\left(\lambda_{1}^{\prime}\right)=\frac{(-1)^{j-1}}{(2 j-1) !} p_{j}
$$

modulo decomposables in $Q\left[p_{1}, \ldots, p_{l}\right]$. But by $[10, \S 1]$ the left hand side is equal to $a\left(\lambda_{1}^{\prime}, j\right) \cdot \tau^{\prime}\left(x_{4 j-1}\right)$. Since

$$
a\left(\lambda_{1}^{\prime}, j\right)=\frac{(-1)^{j-1}}{(2 j-1) !} 2^{q(l, j)}
$$

by Theorem 1, the result follows.

Finally we mention a consequence of this result. Let

$$
\tau: H^{i-1}(\operatorname{Spin}(2 l+1) ; Q) \longrightarrow H^{i}(B \operatorname{Spin}(2 l+1) ; Q)
$$

be the transgression in the Serre spectral sequence of the universal bundle

$$
\operatorname{Spin}(2 l+1) \longrightarrow \operatorname{ESpin}(2 l+1) \longrightarrow B \operatorname{Spin}(2 l+1) .
$$

For $1 \leq j \leq l$ let

$$
y_{4 j} \in H^{4 j}(B \operatorname{Spin}(2 l+1) ; Z)
$$

and

$$
f_{4 j} \in H^{4 j}(B T ; Z)
$$

be as in $[10, \S \S 0$ and 1] (in particular, they are not divisible). Then we can define integers $b(2 j)$ and $c(2 j), 1 \leq j \leq l$, by

$$
\tau\left(x_{4 j-1}\right)=\frac{1}{b(2 j)} \cdot y_{4 j} \quad \text { (modulo decomposables) }
$$

and

$$
(B i) *\left(y_{4 j}\right)=c(2 j) \cdot f_{4 j} \quad(\text { modulo decomposables })
$$


respectively. Since $\tau^{\prime}=(B i) * \tau$, it follows from Proposition 7 that

$$
\frac{c(2 j)}{b(2 j)} f_{4 j}=2^{-q(l, j)} p_{j} \quad \text { (modulo decomposables). }
$$

Thus if $f_{4 j}$ is known, then $b(2 j)$ determines $c(2 j)$ and vice versa.

Let us cite an example. Consider the group $\operatorname{Spin}(11)$, i. e., the case $l=5$. In this case, by (2.3),

$$
H^{*}(\operatorname{Spin}(11) ; Z / 2)=\Delta\left(u_{3}, u_{5}, u_{6}, u_{7}, u_{9}, u_{10}, u\right)
$$

where $\operatorname{deg} u=15 ; S q^{1}\left(u_{5}\right)=u_{6}$ and $S q^{1}\left(u_{9}\right)=u_{10}$. Then by Lemma 2,

$$
\rho\left(x_{3}\right)=u_{3}, \rho\left(x_{7}\right)=u_{7}, \rho\left(x_{11}\right)=u_{5} u_{6}, \rho\left(x_{15}\right)=u \text { and } \rho\left(x_{19}\right)=u_{9} u_{10} .
$$

On the other hand, it follows from Theorem 6.5 of [8] that, in degrees $\leq 20$,

$$
H^{*}(B \operatorname{Spin}(11) ; Z / 2)=Z / 2\left[w_{4}, w_{6}, w_{7}, w_{8}, w_{10}, w_{11}\right] /\left(w_{10} w_{7}+w_{11} w_{6}\right)
$$

where $\operatorname{deg} w_{i}=i ; S q^{1}\left(w_{6}\right)=w_{7}$ and $S q^{1}\left(w_{10}\right)=w_{11}$. By computing Ker $S q^{1} / \operatorname{Im} S q^{1}$, we see that

$$
\rho\left(y_{4}\right)=w_{4}, \rho\left(y_{8}\right)=w_{8}, \rho\left(y_{12}\right)=w_{6}^{2}, \rho\left(y_{16}\right)=w_{6} w_{10} \text { and } \rho\left(y_{20}\right)=w_{10}^{2} .
$$

In the Serre spectral sequence $\left\{E_{r}, d_{r}\right\}$ for the mod 2 cohomology of the fibration (5.1), we find that

$$
\begin{aligned}
& d_{4}\left(1 \otimes u_{3}\right)=w_{4} \otimes 1 \text {; } \\
& d_{8}\left(1 \otimes u_{7}\right)=w_{8} \otimes 1 \text {; } \\
& d_{6}\left(1 \otimes u_{5} u_{6}\right)=w_{6} \otimes u_{6}, \beta_{2}^{F}\left(w_{6} \otimes u_{5}\right)=w_{6} \otimes u_{6}, d_{6}\left(w_{6} \otimes u_{5}\right)=w_{6}^{2} \otimes 1 ; \\
& d_{10}(1 \otimes u)=w_{10} \otimes u_{6}, \beta_{2}^{F}\left(w_{10} \otimes u_{5}\right)=w_{10} \otimes u_{6}, d_{6}\left(w_{10} \otimes u_{5}\right)=w_{6} w_{10} \otimes 1 \text {; } \\
& d_{10}\left(1 \otimes u_{9} u_{10}\right)=w_{10} \otimes u_{10}, \beta_{2}^{F}\left(w_{10} \otimes u_{9}\right)=w_{10} \otimes u_{10}, d_{10}\left(w_{10} \otimes u_{9}\right)=w_{10}^{2} \otimes 1
\end{aligned}
$$

where $\beta_{2}^{F}=1 \otimes S q^{1}$. These facts, together with (2.2), imply that

$$
b(2)=1, b(4)=1, b(6)=2, b(8)=2 \text { and } b(10)=2 \text {. }
$$

On the other hand, modulo decomposables, $f_{4 j}=(1 / 2) p_{j}$ if $j=1,2,4$ and $f_{4 j}=p_{j}$ if $j=3,5$ (for details see $[10, \S 3]$ ). From these results, (5.2) and Theorem 1 it follows that $c(2 j)=1$ for $1 \leq j \leq 5$.

\section{References}

[1] Atiyah, M. F., On the $K$-theory of compact Lie groups, Topology, 4 (1965), 95-99.

[2] Atiyah, M. F. and Hirzebruch, F., Vector bundles and homogeneous spaces, Proc. Sympos. Pure Math., 3, Amer. Math. Soc., 1961.

[3] Borel, A., Sur l'homologie et la cohomologie des groupes de Lie compacts connexes, 
Amer. J. Math., 76 (1954), 273-342.

[4] Hodgkin, L., On the K-theory of Lie groups, Topology, 6 (1967), 1-36.

[5] Husemoller, D., Fibre bundles, Graduate Texts in Math., 20, Springer, 1975.

[6] Ishitoya, K., Kono, A. and Toda, H., Hopf algebra structure of mod 2 cohomology of simple Lie groups, Publ. RIMS, Kyoto Univ., 12 (1976), 141-167.

[7] May, J. P. and Zabrodsky, A., $H^{*} \operatorname{Spin}(n)$ as a Hopf algebra, J. Pure Appl. Algebra, 10 (1977), 193-200.

[8] Quillen, D., The mod 2 cohomology rings of extra-special 2-groups and the spinor groups, Math. Ann., 194 (1971), 197-212.

[9] Steenrod, N., The topology of fibre bundles, Princeton Math. Ser, 14, Princeton Univ. Press, 1951.

[10] Watanabe, T., Chern characters on compact Lie groups of low rank, Osaka J. Math., 22 (1985), 463-488.

[11] Yokota, I., Groups and representations, Shōkabō, 1973 (in Japanese). 
